\title{
Two-goal Local Search and Inference Rules for Minimum Dominating Set
}

\author{
Shaowei Cai ${ }^{1,2 *}$, Wenying Hou ${ }^{3}$, Yiyuan Wang ${ }^{4}$, Chuan $\mathbf{L u o}^{5}$ and Qingwei Lin ${ }^{5}$ \\ ${ }^{1}$ State Key Laboratory of Computer Science, Institute of Software, Chinese Academy of Sciences, China \\ ${ }^{2}$ School of Computer Science and Technology, University of Chinese Academy of Sciences, China \\ ${ }^{3}$ School of Software and Microelectronics, Peking University, China \\ ${ }^{4}$ School of Computer Science and Information Technology, Northeast Normal University, China \\ ${ }^{5}$ Microsoft Research, China \\ caisw@ios.ac.cn, houwyvenny@pku.edu.cn, yiyuanwangjlu@126.com
}

\begin{abstract}
Minimum dominating set (MinDS) is a canonical NP-hard combinatorial optimization problem with applications. For large and hard instances one must resort to heuristic approaches to obtain good solutions within reasonable time. This paper develops an efficient local search algorithm for MinDS, which has two main ideas. The first one is a novel local search framework, while the second is a construction procedure with inference rules. Our algorithm named FastDS is evaluated on 4 standard benchmarks and 3 massive graphs benchmarks. FastDS obtains the best performance for almost all benchmarks, and obtains better solutions than stateof-the-art algorithms on massive graphs.
\end{abstract}

\section{Introduction}

Given an undirected graph $G=(V, E)$, a dominating set $D$ is a subset of vertices such that each vertex in $V \backslash D$ has at least one adjacent vertex in $D$. Dominating set is an important concept in graph theory, and have applications in diverse areas, especially in social networks [Wang et al., 2009; Chalupa, 2018]. One typical problem is to find a smallest group of influential individuals or a set of initial seeds in a social network, so that all participants can be reached with only one hop from the seeds. This problem is equivalent to finding a minimum dominating set (MinDS) for the network.

The MinDS problem is a classic NP-hard problem, and cannot be approximated with a constant ratio under the assumption $P \neq N P$ [Raz and Safra, 1997]. Moreover, negative results have been proved for the approximation of MinDS even when limited to power law graphs [Gast et al., 2015]. A number of works have been done on exact algorithms for MinDS, which mainly focus on improving the upper bound of running time. State-of-the-art exact algorithms for MinDS are based on the branch and reduce paradigm and can achieve a run time of $O\left(1.4969^{n}\right)$ [van Rooij and Bodlaender, 2011]. Fixed parameterized algorithms have allowed to obtain better complexity results [Karthik C. S. et al., 2018]. The main focus of such algorithms is on theoretical aspects.

\footnotetext{
${ }^{*}$ Corresponding author
}

In practice, one usually resorts to heuristic approaches to obtain good solutions within reasonable time. Although greedy algorithms are fast, the obtained dominating sets are far from satisfactory. A comparison among several greedy heuristics for MinDS can be found in [Sanchis, 2002]. Heuristic search algorithms usually return very good or even optimal solutions within a reasonable time budget. Commonly used heuristic search methods, including genetic algorithm [Hedar and Ismail, 2010] and ant colony optimization [Jovanovic et al., 2010; Potluri and Singh, 2011; Potluri and Singh, 2013], have been developed to solve MinDS. Hyper meta-heuristic algorithms combine different heuristic search algorithms and preprocessing techniques to obtain better performance [Potluri and Singh, 2013; Chaurasia and Singh, 2015; Bouamama and Blum, 2016; Lin et al., 2016; Abu-Khzam et al., 2017]. These algorithms were tested on standard benchmarks with up to thousand vertices. Recently, the configuration checking (CC) strategy [Cai et al., 2011] has been applied to MinDS and led to two local search algorithms. Wang et al. proposed the $\mathrm{CC}^{2} \mathrm{FS}$ algorithm for both unweighted and weighted MinDS [Wang et al., 2017], and obtained better solutions than ACO-PP-LS [Potluri and Singh, 2013] on standard benchmarks. Afterwards, another CC-based local search named FastMWDS was proposed, which significantly improved $\mathrm{CC}^{2} \mathrm{FS}$ on weighted massive graphs [Wang et al., 2018]. Chalupa proposed an orderbased randomised local search named RLS $_{o}$ [Chalupa, 2018], and achieved better results than ACO-LS and ACO-PP-LS [Potluri and Singh, 2011; Potluri and Singh, 2013] on standard benchmarks of unit disk graphs as well as some massive graphs. Very recently, Fan et. al. designed a local search algorithm named ScBppw [Fan et al., 2019], based on two ideas including score checking and probabilistic random walk.

This work develops an efficient and robust algorithm for MinDS named FastDS, which achieves best results on almost all standard and massive benchmarks, and significantly outperforms state of the art algorithms on massive benchmarks. There are two main novel ideas in FastDS. The first one is a new local search framework. A typical local search framework for MinDS, is to solve the problem by iteratively solving the $k$-DS problem - given an integer $k$, searching for a $k$ sized dominating set [Fan et al., 2019]. Specifically, when finding a $k$ sized feasible solution, the algorithm removes one vertex and goes on to search for a $(k-1)$ size solution, by 
exchanging a pair of vertices in each step. We propose a new local search framework. When finding a $k$ size feasible solution, our algorithm goes on to search for a solution with $(k-1)$ or $(k-2)$ size. That is, our algorithm targets two goals during the search. This accelerates the convergence speed, and also expands the search region.

Moreover, we propose inference rules for MinDS, which are used to fix vertices that must be in some optimal solution. The inference rules are particularly useful for solving massive graphs. In order to solve combinatorial optimization problems on massive graphs, reduction rules have been implemented to reduce the size of the graphs. Examples include maximum clique [Cai and Lin, 2016], vertex cover [Cai et al., 2017], k-plex [Gao et al., 2018], as well as the weighted dominating set problem [Wang et al., 2018]. Unfortunately, reduction rules become very restricted when coming to the MinDS problem, due to the special feature of the problem. For example, even if we can fix the value of a vertex to be 1 (being selected), we cannot delete it from the graph, as this vertex may be responsible for dominating other vertices in the remaining graph. Although there are some reduction rules for minimum weighted dominating set, they are based on the differentials among vertex weights [Wang et al., 2018], and cannot be applied to MinDS as all vertices have the same weight there. We propose a compromise solution to design inference rules rather than reduction rules. We fix the value of some vertices using inference, without deleting them.

We conduct extensive experiments to compare FastDS with state-of-the-art heuristic algorithms on a broad range of benchmarks, including 4 standard benchmarks and 3 massive graphs benchmarks. FastDS obtains the best results for almost all benchmarks, and significantly outperforms state-ofthe-art algorithms on the 3 massive benchmarks.

The paper is organized as follows. Section 2 presents basic definitions. Section 3 introduces the new local search framework. Sections 4 presents the inference rules. Section 5 describes our FastDS algorithm. Experimental results are shown in Section 6 and Section 7 gives concluding remarks.

\section{Preliminaries}

An undirected graph $G=(V, E)$ consists of a vertex set $V$ and an edge set $E$. Each edge $e=(v, u)$ connects two vertices $v$ and $u$, which are also called endpoints of $e$. Two vertices are neighbors if they belong to a same edge. For a vertex $v, N(v)=\{u \in V \mid(u, v) \in E\}$ is the set of neighbors of $v$, and the degree of $v$ is degree $(v)=|N(v)|$. The closed neighborhood of $v$ is $N[v]=N(v) \cup\{v\}$. Also, we use $N^{2}[v]$ to denote the set of $v$ 's closed neighborhood and its neighbors' neighbors, i.e., $N^{2}[v]=N[v] \cup\left(\bigcup_{u \in N(v)} N(u)\right)$. For a vertex set $S \subseteq V$, we use $N[S]=\bigcup_{v \in S} N[v]$ to denote the closed neighborhood of $S$.

A candidate solution (also known as partial solution) for MinDS is a subset of $V$. Given a candidate solution $D$, the value of a vertex $v$, denoted as value $(v)$, indicates whether it is selected in the candidate solution or not, which is 1 if $v \in$ $D$, and 0 otherwise. A vertex $v$ is dominated by a candidate solution $D$ if $v \in N[D]$, and is non-dominated otherwise. The cost of a candidate solution $D$, denoted as $\operatorname{cost}(D)$, is defined as the number of non-dominated vertices under $D$. A commonly used scoring function for MinDS is defined as

$$
\operatorname{score}(v)=\operatorname{cost}(D)-\operatorname{cost}\left(D^{\prime}\right)
$$

where $D^{\prime}=D \backslash\{v\}$ if $v \in D$, and $D^{\prime}=D \cup\{v\}$ otherwise. Obviously, $\operatorname{score}(v) \leq 0$ if $v \in D$, and $\operatorname{score}(v) \geq 0$ if $v \notin D$.

We use $D$ to denote the current candidate solution, i.e., the set of vertices currently selected for dominating, and $U$ to denote the set of non-dominated vertices. $D^{*}$ is the best solution found so far, and $D^{*}$ at the end of the algorithm is returned by the algorithm. The age of a vertex $v$ is the number of steps that have occurred since $v$ last changed its state.

\section{Two-goal Local Search}

We propose a novel local search framework for MinDS, which allows the algorithm to target two different sizes during each period of the search.

For subset problems whose goal is to find a minimum set satisfying given constraints, such as MinDS, minimum vertex cover (MinVC) and minimum set cover, a popular paradigm of local search is to iteratively find a feasible solution of a fixed size [Musliu, 2006; Richter et al., 2007; Cai et al., 2013; Gao et al., 2015; Fan et al., 2019; Li et al., 2019]. Specifically, when finding a $k$-sized solution, the algorithm focuses on searching for a $(k-1)$-sized solution by fixing the size of the partial solution to be $k-1$ at the end of each step. To this end, a popular local search framework is the two-stage exchanging framework [Cai et al., 2013], where one vertex is removed from the partial solution in the first stage and another vertex is added in the second stage. Because of its good performance and low complexity, the two-stage exchanging framework has been applied to local search algorithms for the MinDS problem [Fan et al., 2019].

We propose a new local search framework for MinDS (Algorithm 1). The local search starts from an initial feasible solution (line 2) and searches for a feasible solution as small as possible. Specifically, when $D$ becomes a feasible solution, $D^{*}$ is updated (line 5) and then a vertex is removed from $D$ (line 6). When $D$ is not a feasible solution, the algorithm iteratively exchanges vertices until $D$ becomes a new dominating set. It first removes vertices to make $|D|=\left|D^{*}\right|-3$; in the adding phase, one vertex is added to $D$, and after that, if $D$ is still infeasible, another vertex is added with a certain probability. Thus, the size of $D$ can be $\left|D^{*}\right|-1$ or $\left|D^{*}\right|-2$ at the end of a search step. Finally, the best found dominating set $D^{*}$ is returned when the time limit is reached.

Previous local search algorithms focus on seeking a $(k-1)$ sized solution after finding a $k$-sized solution. Differently, our framework searches for a $(k-1)$-sized or $(k-2)$-sized solution after it finds a $k$-sized solution. This has two advantages. (1) It accelerates the converge of the search, as the algorithm sometimes directly finds a $(k-2)$-sized solution without finding a $(k-1)$-sized one. Note that if we set the goal to be $(k-2)$ after finding a $k$-sized solution, we would fail to find the optimal solution if its size is $(k-1)$. Our framework with two goals can improve the search efficiency without losing the ability to find the optimal solution. (2) It expands the search region for 


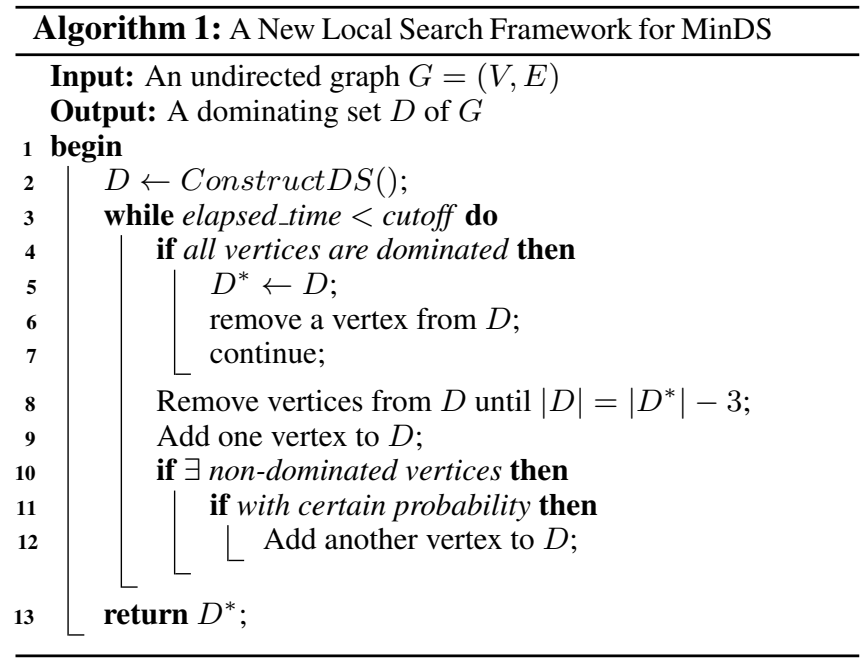

the adding stage by keeping a smaller candidate solution at the end of the removing stage. A smaller candidate solution leads to a larger $N[U]$ ( $U$ is the set of non-dominated vertices), which implies more candidate adding vertices in the adding stage. Finally, we note that our local search framework can be applied to other subset problems.

\section{Inference Rules for MinDS}

To improve the performance of local search for MinDS on large instances, we propose three inference rules, which can fix a considerable portion of the vertices for massive graphs.

Isolated vertex rule: If a vertex $v$ is not adjacent to any other vertices, or all its neighbors have a fixed value 0 (not selected), then $v$ 's value is fixed to 1 .

This rule is obvious: in order to make the vertex $v$ dominated, there is only one option - including $v$ in the solution.

Leaf rule: If a vertex $v$ is adjacent to only one vertex $u$, or all its neighbors but $u$ have a fixed value 0 , then value $(u)$ is fixed to 1 and value $(v)$ is fixed to 0 .

The reasoning of this rule is as follows. In order to dominate vertex $v$, at least one of $u$ and $v$ should be selected, and we prove that the best way to do so is to choose $u$ and not choose $v$. (1) If both $u$ and $v$ are selected in a dominating set $D$, then $D \backslash\{v\}$ is a smaller dominating set; (2) If $v$ is selected and $u$ is not, i.e., $v \in D$ and $u \notin D$, then we can exchange $u$ and $v$ and the resulting set $D \backslash\{v\} \cup\{u\}$ is still a dominating set, as removing $v$ can only have impact on vertices $u$ and $v$, and by adding $v$ we dominate both of them.

Triangle rule: For a triangle $\{u, v, w\}$ in the graph, where $N(u)=\{v, w\}, N(v)=\{u, w\}$ and $N(w) \supset\{u, v\}$, value $(w)$ is fixed to 1 while value $(u)$ and value $(v)$ are fixed to 0 .

The reasoning follows as this: For any dominating set $D$, in order to dominate vertices $u$ and $v$, at least one vertex from $\{u, v, w\}$ is in $D$. Now, since $N[u]=N[v]=\{u, v, w\} \subset$ $N[w]$, we know that, any vertex that can be dominated by $u$ and $v$ can be dominated by $w$, but not vice versa.

We can generalize the Triangle rule as follows. However, due to the high computation cost, the generalized rule is not implemented in our algorithm.

Generalized Triangle rule: For a vertex $w$ and a vertex set $S \subseteq N(w)$, if $N[S] \subset N[w]$, then value $(w)$ is fixed to 1 , and the value of any vertex in $S$ is fixed to 0 .

\section{The FastDS Algorithm}

We develop a local search algorithm for MinDS named FastDS (Algorithm 3), which is based on our two-goal framework and employs inference rules.

\subsection{Initial Construction}

In the beginning, the algorithm constructs an initial solution by a greedy construction procedure, making use of the inference rules. A key concept of the algorithm is the nondominated neighborhood and non-dominated degree.

Definition 1. Given a graph $G=(V, E)$ and a vertex subset $D$, the non-dominated neighborhood of a vertex $v$ is $N_{r}[v]=$ $\{u \mid u \in N[v], N[u] \cap D=\emptyset\}$, and the non-dominated degree of $v$ is degree de $_{r}(v)=\left|N_{r}[v]\right|$.

Intuitively, the set $N_{r}[v]$ includes all the vertices that are currently non-dominated and would be dominated if $v$ is added to the candidate solution $D$. Thus, $\operatorname{degree}_{r}(v)$ measures the benefits of adding vertex $v$.

The construction procedure fist traverses all vertices and employs inference rules to fix the vertices satisfying at least one of the rules. Then, it utilizes a greedy strategy based on non-dominated degrees to iteratively add vertices until a dominating set is obtained. At the end of the construction, redundant vertices are removed from $D$ to make it minimal.

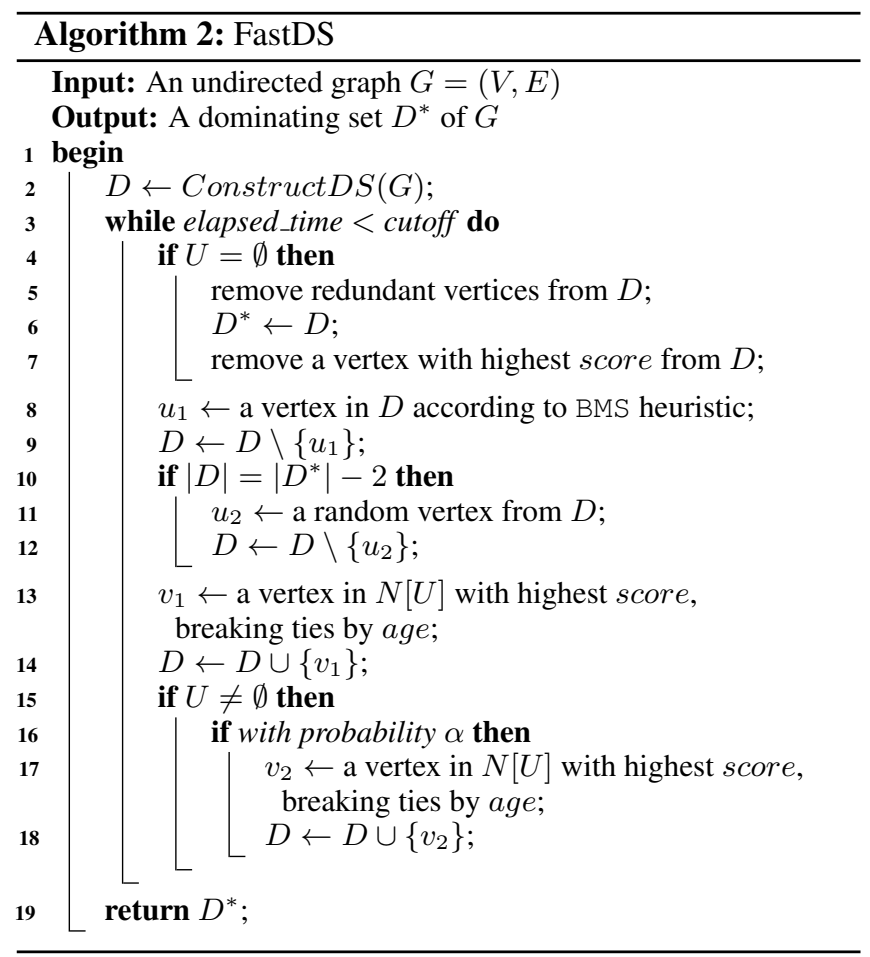




\subsection{Local Search}

The constructed initial solution is then fed to the local search phase for improving. During the local search process, whenever $D$ becomes a feasible solution, redundant vertices (if any) are first removed from $D$, and $D^{*}$ is updated to $D$. After that, a vertex with the maximum score (i.e., the minimum loss) is removed. Then, the local search iteratively executes exchanging steps until $D$ becomes a feasible solution.

Each exchanging step consists of a removing stage and an adding stage. In the removing stage (lines 8-12), one or two vertices are removed from $D$. The first vertex $u_{1}$ is selected from $D$ according to a sampling heuristic named Best from Multiple Selections (BMS) [Cai, 2015], which randomly samples $t$ vertices in $D$ and picks the one with the maximum score to remove ( $t$ is suggested near 50 [Cai, 2015] and is set to 45 in this work). If there is more than one such vertex, the vertex with the largest age is selected to remove. Additionally, after $u_{1}$ is removed from $D$, if $\left|D^{*}\right|-|D|$ equals 2 rather than 3 , one more vertex is removed, to ensure $|D|=\left|D^{*}\right|-3$ at the end of the removing stage.

In the adding stage (lines 13-18), one or two vertices are added into $D$. First, a vertex $v_{1} \in N[U]$ ( $U$ is the set of nondominated vertices) with the highest score is added to $D$, breaking ties by preferring the one with largest $a g e^{1}$. Then, if there are non-dominated vertices, with a probability of $\alpha$, a second vertex $v_{2}$ is selected in the same way as the first one and added to $D$.

An important implementation detail is that, when removing or adding a vertex $x$, the score of vertices in $N^{2}[x]$ may change, and should be updated accordingly.

\section{Experiments}

We evaluate FastDS on 7 benchmarks, including 4 standard benchmarks in the literatures and 3 massive benchmarks.

UDG: This is a widely used standard benchmark for MinDS [Hedar and Ismail, 2010; Potluri and Singh, 2011; Potluri and Singh, 2013; Chalupa, 2018]. UDG contains 120 instances generated by the topology generator ${ }^{2}$. UDG is divided into 12 families, each of which has 10 instances.

T1: This data set consists of 520 instances where each instance has two different weight functions [Jovanovic et al., 2010]. We select these original graphs where the weight of each vertex is set to 1 . There are 52 families, each of which contains 10 instances with the same size. To save space, we do not report the results on graphs with 50 vertices.

DIMACS: This benchmark is from the Second DIMACS Implementation Challenge (1992-1993) ${ }^{3}$, including problems from real applications and randomly generated graphs.

BHOSLIB: This benchmark are generated based on the RB model [Xu et al., 2007] near the phase transition. It is a popular benchmark for graph theoretic problems.

\footnotetext{
${ }^{1}$ In practice, acceleration can be gained for the classic benchmarks by picking a random non-dominated vertex with a small probability such as 0.005 here.

${ }^{2} \mathrm{http}: / / \mathrm{www} . \mathrm{michelemastrogiovanni.net/software/download/}$

${ }^{3} \mathrm{ftp} / / /$ dimacs.rutgers.edu/pub/challenges
}

\begin{tabular}{|c|c|c|c|c|c|}
\hline Instance & $\mathrm{CC}^{2} \mathrm{FS}$ & FastMWDS & $\mathrm{RLS}_{o}$ & ScBppw & FastDs \\
\hline Family & $\overline{D_{\min }}$ & $\overline{D_{\min }}$ & $\overline{D_{\min }}$ & $\overline{D_{\min }}$ & $\overline{D_{\min }}$ \\
\hline V100U150 & 17 & 17 & 17 & 17.3 & 17 \\
\hline V100U200 & 10.4 & 10.4 & 10.4 & 10.6 & 10.4 \\
\hline V250U150 & 18 & 18 & 18 & 19 & 18 \\
\hline V250U200 & 10.8 & 10.8 & 10.8 & 11.5 & 10.8 \\
\hline V500U150 & 18.5 & 18.5 & 18.6 & 20.1 & 18.5 \\
\hline V500U200 & 11.2 & 11.2 & 11.2 & 12.4 & 11.2 \\
\hline V50U150 & 12.9 & 12.9 & 12.9 & 12.9 & 12.9 \\
\hline V50U200 & 9.4 & 9.4 & 9.4 & 9.4 & 9.4 \\
\hline V800U150 & 19 & 19 & 19.1 & 20.9 & 19 \\
\hline V800U200 & 11.7 & 11.7 & 11.9 & 12.6 & 11.8 \\
\hline V1000U150 & 19.1 & 19.1 & 19.2 & 21.3 & 19.1 \\
\hline V1000U200 & 12 & 12 & 12 & 13 & 12 \\
\hline
\end{tabular}

Table 1: Experiment results on UDG benchmark

\begin{tabular}{|c|c|c|c|c|c|c|c|c|c|c|c|}
\hline Instance & $\mathrm{CC}^{2} \mathrm{FS}$ & MWDS & $\mathrm{RLS}_{0}$ & ScBpw & FastDS & Instance & $\mathrm{CC}^{2} \mathrm{FS}$ & MWDS & $\mathrm{RLS}_{0}$ & ScBpw & FastDS \\
\hline Family & $\overline{D_{\min }}$ & $\overline{D_{\min }}$ & $\overline{D_{\min }}$ & $\overline{D_{\min }}$ & $\overline{D_{\min }}$ & Family & $\overline{D_{\min }}$ & $\overline{D_{\min }}$ & $\overline{D_{\min }}$ & $\overline{D_{\min }}$ & $\overline{D_{\min }}$ \\
\hline V100E100 & 33.6 & 33.6 & 33.6 & 33.6 & 33.6 & V250E5000 & 11 & 11 & 11.2 & 11.5 & \\
\hline V100E1000 & 7.5 & 7.5 & 7.5 & 7.8 & 7.5 & V250E750 & 44 & 44 & 44.3 & 44.9 & 44 \\
\hline V100E2000 & 4.1 & 4.1 & 4.1 & 4.3 & 4.1 & V300E1000 & 48.6 & 48.6 & 49.1 & 49.5 & 48.6 \\
\hline V100E250 & 19.9 & 19.9 & 19.9 & 20.1 & 19.9 & V300E2000 & 29.4 & 29.4 & 30 & 30.6 & 29.4 \\
\hline V100E500 & 12.2 & 12.2 & 12.2 & 12.5 & 12.2 & V300E300 & 100 & 100 & 100 & 100.1 & 100 \\
\hline V100E750 & 9 & 9 & 9 & 9.5 & 9 & V300E3000 & 22 & 22 & 22.8 & 22.9 & 22 \\
\hline V150E1000 & 15 & 15 & 15.1 & 15.4 & 15 & V300E500 & 77.7 & 77.7 & 77.8 & 78.4 & 77.7 \\
\hline V150E150 & 50 & 50 & 50 & 50 & 50 & V300E5000 & 15.1 & 15.1 & 15.7 & 15.9 & 15.1 \\
\hline V150E2000 & 9 & 9 & 9 & 9.5 & 9 & V300E750 & 59.6 & 59.6 & 59.6 & 60 & 59.6 \\
\hline V150E250 & 39.1 & 39.1 & 39.3 & 39.3 & 39.1 & V500E1000 & 114.7 & 114.7 & 114.8 & 116.3 & 114.7 \\
\hline V150E3000 & 6.9 & 6.9 & 6.9 & 6.9 & 6.9 & V500E 10000 & 22 & 22.1 & 23.2 & 23.3 & 22.2 \\
\hline V150E500 & 24.6 & 24.6 & 24.7 & 24.9 & 24.6 & V500E2000 & 71.2 & 71.2 & 72.5 & 72.9 & 71.2 \\
\hline V150E750 & 18.3 & 18.3 & 18.5 & 18.8 & 18.3 & V500E500 & 167 & 167 & 167 & 167 & 167 \\
\hline V200E1000 & 24.4 & 24.4 & 24.7 & 25 & 24.4 & V500E5000 & 36.9 & 36.9 & 38.4 & 38.7 & 36.9 \\
\hline V200E2000 & 15 & 15 & 15 & 15.3 & 15 & V800E100 & 267 & 267 & 267 & 267 & 267 \\
\hline V200E250 & 61.1 & 61.1 & 61.1 & 61.7 & 61.1 & V800E1000 & 242.5 & 242.5 & 242.5 & 246.5 & 242.5 \\
\hline V200E3000 & 11 & 11 & 11.1 & 11.3 & 11 & V800E 10000 & 50.4 & 50.3 & 52.7 & 53.1 & 50.2 \\
\hline V200E500 & 39.6 & 39.6 & 39.6 & 40 & 39.6 & V800E2000 & 158.3 & 158.3 & 159.4 & 162.1 & 158.3 \\
\hline V200E750 & 30 & 30 & 30.1 & 30.4 & 30 & V800E5000 & 82.6 & 82.6 & 85.9 & 86.3 & 82.6 \\
\hline V250E 1000 & 36 & 36 & 36.3 & 36.6 & 36 & V1000E1000 & 333.7 & 334 & 333.7 & 333.7 & 333.7 \\
\hline V250E2000 & 21.6 & 21.6 & 22.1 & 22.4 & 21.6 & V1000E 10000 & 74.2 & 74.1 & 77.8 & 77.6 & 74 \\
\hline V250E 250 & 83.3 & 83.3 & 83.3 & 83.3 & 83.3 & V1000E15000 & 55.5 & 55.1 & 58.2 & 58.2 & 55 \\
\hline $\mathrm{V} 250 \mathrm{E} 3000$ & 16 & 16 & 16.5 & 16.6 & 16 & V1000E20000 & 45.2 & 45.4 & 47.1 & 47.4 & 45 \\
\hline V250E500 & 57.8 & 57.8 & 57.9 & 58.1 & 57.8 & V1000E5000 & 121.2 & 121.1 & 125.3 & 126 & 121.1 \\
\hline
\end{tabular}

Table 2: Experiment results on T1 benchmark. To save space, we denote FastMWDS as MWDS.

SNAP: This benchmark is from Stanford Large Network Dataset Collection ${ }^{4}$. It is a collection of real world graphs from $10^{4}$ vertices to $10^{7}$ vertices.

DIMACS10: This benchmark is from the 10th DIMACS implementation challenge $(2010)^{5}$, which aims to provide large challenging instances for graph theoretic problems.

Network Repository: The Network Data Repository [Rossi and Ahmed, 2015] collects massive graphs from various areas. Many of the graphs have 100 thousands or millions of vertices. This benchmark has been widely used for graph theoretic problems including vertex cover, clique, coloring, and dominating set problems.

For SNAP and DIMACS10 benchmarks, as in previous literatures [Lin et al., 2017; Verma et al., 2015], we only report the results on graphs with at least 30000 vertices. For Repository benchmark, we choose the graphs with at least $10^{5}$ vertices. This results in 22, 31 and 65 graphs in SNAP, DIMACS10, and Repository, respectively.

\subsection{Experiment Setup}

FastDS is implemented in C++ and complied by g++ with 'O3' option, and the parameter $\alpha$ is set to 0.6. All experiments are run on a server with Intel Xeon E5-2640 v4 2.40GHz with 128GB RAM under CentOS 7.5.

\footnotetext{
${ }^{4}$ http://snap.stanford.edu/data

${ }^{5}$ https://www.cc.gatech.edu/dimacs10/
} 


\begin{tabular}{llllll}
\hline & CC ${ }^{2} \mathrm{FS}$ & FastMWDS & $\mathrm{RLS}$ & $\mathrm{ScBppw}$ & FastDS \\
Instance & $D_{\min }\left(D_{a v g}\right)$ & $D_{\min }\left(D_{a v g}\right)$ & $D_{\min }\left(D_{a v g}\right)$ & $D_{\min }\left(D_{a v g}\right)$ & $D_{\min }\left(D_{a v g}\right)$ \\
\hline frb40-19-1 & $\mathbf{1 4}$ & $\mathbf{1 4}$ & $14(15)$ & $15(15.8)$ & $\mathbf{1 4}$ \\
frb40-19-2 & $\mathbf{1 4}$ & $\mathbf{1 4}$ & $15(15.4)$ & $16(16.5)$ & $\mathbf{1 4}(14.3)$ \\
frb40-19-3 & $14(14.8)$ & $\mathbf{1 4}(\mathbf{1 4 . 6})$ & $15(15.6)$ & $16(16.5)$ & $14(14.7)$ \\
frb40-19-4 & $\mathbf{1 4}$ & $\mathbf{1 4}$ & $15(15.8)$ & $15(16.2)$ & $\mathbf{1 4}$ \\
frb40-19-5 & $\mathbf{1 4}$ & $\mathbf{1 4}$ & $15(15.1)$ & $15(15.2)$ & $\mathbf{1 4}$ \\
frb45-21-1 & $\mathbf{1 6}$ & $\mathbf{1 6}$ & $16(17.4)$ & $17(18)$ & $\mathbf{1 6}$ \\
frb45-21-2 & $16(16.2)$ & $\mathbf{1 6}$ & $17(17.8)$ & $18(18.1)$ & $\mathbf{1 6}$ \\
frb45-21-3 & $\mathbf{1 6}$ & $\mathbf{1 6}$ & $17(17.5)$ & $17(17.8)$ & $\mathbf{1 6}$ \\
frb45-21-4 & $\mathbf{1 6}$ & $\mathbf{1 6}$ & $17(17.8)$ & $17(18)$ & $\mathbf{1 6}$ \\
frb45-21-5 & $\mathbf{1 6}$ & $\mathbf{1 6}$ & $17(17.7)$ & $18(18.2)$ & $\mathbf{1 6}$ \\
frb50-23-1 & $\mathbf{1 8}$ & $\mathbf{1 8}$ & $19(19.6)$ & $19(19.9)$ & $\mathbf{1 8}$ \\
frb50-23-2 & $\mathbf{1 8}$ & $\mathbf{1 8}$ & $19(20.1)$ & $19(19.8)$ & $\mathbf{1 8}$ \\
frb50-23-3 & $\mathbf{1 8}$ & $\mathbf{1 8}$ & $19(20)$ & 20 & $\mathbf{1 8}$ \\
frb50-23-4 & $\mathbf{1 8}$ & $\mathbf{1 8}$ & $20(20.1)$ & $19(20)$ & $\mathbf{1 8}$ \\
frb50-23-5 & $\mathbf{1 8}$ & $\mathbf{1 8}$ & $19(19.8)$ & $19(19.9)$ & $\mathbf{1 8}$ \\
frb53-24-1 & $\mathbf{1 9}$ & $\mathbf{1 9}$ & $20(21.1)$ & $21(21.1)$ & $\mathbf{1 9}$ \\
frb53-24-2 & $\mathbf{1 9}$ & $19(19.1)$ & $20(21.4)$ & $21(21.2)$ & $\mathbf{1 9}$ \\
frb53-24-3 & $\mathbf{1 9}$ & $\mathbf{1 9}$ & $20(20.5)$ & $20(20.4)$ & $\mathbf{1 9}$ \\
frb53-24-4 & $\mathbf{1 8}(\mathbf{1 8 . 7})$ & 19 & $19(20)$ & $19(20.2)$ & $\mathbf{1 8}(\mathbf{1 8 . 7})$ \\
frb53-24-5 & $19(19.1)$ & $\mathbf{1 9}$ & $20(21.3)$ & $20(21.5)$ & $\mathbf{1 9}$ \\
frb56-25-1 & $\mathbf{2 0}$ & $\mathbf{2 0}$ & $21(21.8)$ & $21(22.2)$ & $\mathbf{2 0}$ \\
frb56-25-2 & $\mathbf{2 0}$ & $20(20.1)$ & $21(22.2)$ & $21(22.5)$ & $\mathbf{2 0}$ \\
frb56-25-3 & $\mathbf{2 0}$ & $\mathbf{2 0}$ & $22(22.7)$ & $21(22.2)$ & $\mathbf{2 0}$ \\
frb56-25-4 & $20(20.5)$ & $20(20.9)$ & $22(22.7)$ & $22(22.5)$ & $\mathbf{2 0}$ \\
frb56-25-5 & $\mathbf{2 0}$ & $\mathbf{2 0}$ & $21(21.5)$ & $21(21.8)$ & $\mathbf{2 0}$ \\
frb59-26-1 & 21 & 21 & $22(23)$ & $22(22.6)$ & $\mathbf{2 0}(\mathbf{2 0 . 5})$ \\
frb59-26-2 & $\mathbf{2 1}$ & $\mathbf{2 1}$ & $22(22.8)$ & $22(22.6)$ & $\mathbf{2 1}$ \\
frb59-26-3 & $21(21.7)$ & $\mathbf{2 1}(21.5)$ & $22(23.1)$ & $23(23.1)$ & $\mathbf{2 1}$ \\
frb59-26-4 & $21(21.1)$ & $\mathbf{2 1}(21.1)$ & $23(23.6)$ & $23(23.6)$ & $\mathbf{2 1}$ \\
frb59-26-5 & $21(21.9)$ & $21(21.8)$ & $24(24.3)$ & $23(24)$ & $\mathbf{2 1}$ \\
frb100-40 & $37(37.9)$ & $38(38.1)$ & $40(40.7)$ & $39(40.1)$ & $\mathbf{3 6}(\mathbf{3 6 . 4})$ \\
\hline & & & & &
\end{tabular}

Table 3: Experiment results on BHOSLIB benchmark

\begin{tabular}{llll}
\hline Benchmark & CC $^{2} \mathrm{FS}$ & FastMWDS & FastDS \\
\hline UDG & $0.21 \mathrm{~s}$ & $0.83 \mathrm{~s}$ & $22.19 \mathrm{~s}$ \\
T1 & $4.88 \mathrm{~s}$ & $8.35 \mathrm{~s}$ & $11.23 \mathrm{~s}$ \\
BHOSLIB & $96.68 \mathrm{~s}$ & $101.44 \mathrm{~s}$ & $95.62 \mathrm{~s}$ \\
\hline
\end{tabular}

Table 4: Averaged run time on standard benchmarks

We compare FastDS with four state-of-the-art heuristic algorithms, including $\mathrm{CC}^{2} \mathrm{FS}$ [Wang et al., 2017], FastMWDS [Wang et al., 2018], RLS [Chalupa, 2018] and ScBppw [Fan et al., 2019]. CC ${ }^{2} \mathrm{FS}$ is good at solving standard benchmarks, while FastMWDS and ScBppw are designed to solve massive graphs, and $\mathrm{RLS}_{o}$ is a recent algorithm that outperforms previous ant optimization and hyper meta-heuristic algorithms. The codes of $\mathrm{CC}^{2} \mathrm{FS}$, FastMWDS and $\mathrm{RLS}_{o}$ were kindly provided by their authors while the codes of ScBppw were open online $^{6}$. All of them are implemented in $\mathrm{C}++$ and complied by g++ with '-O3' option.

For each instance, all algorithms are executed 10 times with random seeds $1,2,3 \ldots 10$. The time limit of each run is 1000 seconds. For each instance, we report the best size $\left(D_{\text {min }}\right)$ and the average size $\left(D_{\text {avg }}\right)$ of the solutions found over the 10 runs. When $D_{\text {avg }}=D_{\text {min }}$, we do not report $D_{\text {avg. }}$ For UDG and T1, we report for each family the averaged value of $D_{\min }$, denoted as $\overline{D_{\min }}$. When failing to find a solution in time limit, the cell is marked as 'N/A'.

\subsection{Results on Standard Benchmarks}

Results on UDG, T1 and BHOSLIB benchmarks are reported in Tables 1, 2 and 3 respectively. The DIMACS instances are so easy that $\mathrm{CC}^{2} \mathrm{FS}$, FastMWDS and FastDS find the

\footnotetext{
${ }^{6}$ https://github.com/Fan-Yi/
}

\begin{tabular}{|c|c|c|c|c|}
\hline Instance & $\begin{array}{l}\text { FastMWDS } \\
D_{\min }\left(D_{a v g}\right)\end{array}$ & $\begin{array}{l}\operatorname{RLS}_{0} \\
D_{\text {min }}\left(D_{\text {avg }}\right)\end{array}$ & $\begin{array}{l}\text { ScBppw } \\
D_{\min }\left(D_{\text {avg }}\right)\end{array}$ & $\begin{array}{l}\text { FastDS } \\
D_{\min }\left(D_{a v g}\right)\end{array}$ \\
\hline Amazon0302 & $35616(35619.7)$ & $38735(38771.1)$ & $36199(36212.5)$ & $35593(35602.7)$ \\
\hline Amazon0312 & 45531(45538) & $49326(49362.7)$ & 45914(45931.9) & $45490(45493.7)$ \\
\hline Amazon0505 & $47362(47374.6)$ & $51281(51306.9)$ & 47734(47748.4) & $47310(47317.7)$ \\
\hline Amazon0601 & $42319(42325.5)$ & $45952(45989.9)$ & $42717(42732.8)$ & $42289(42294.2)$ \\
\hline Cit-HepPh & 3078(3078.6) & 3192(3209.3) & $3087(3088.9)$ & $\mathbf{3 0 7 8}(3078.9)$ \\
\hline Cit-HepTh & 2935(2935.7) & 2985(2993.4) & 2944(2948.6) & 2936(2936.1) \\
\hline cit-Patents & $650610(651187.7)$ & $667418(667594.3)$ & $622886(622999.5)$ & $621722(621742.3)$ \\
\hline Email-EuAll & 18181 & 18181(18181.6) & 18181 & 18181 \\
\hline p2p-Gnutela04 & 2227 & 2227 & 2227 & 2227 \\
\hline p2p-Gnutela24 & $5418(5418)$ & $\mathbf{5 4 1 8}(5418.5)$ & 5418 & 5418 \\
\hline p2p-Gnutela25 & 4519 & $\mathbf{4 5 1 9}(4519.1)$ & 4519 & 4519 \\
\hline p2p-Gnutela30 & 7169 & 7169(7169.4) & $\mathbf{7 1 6 9}(7169.2)$ & 7169 \\
\hline p2p-Gnutela31 & 12582 & $12593(12596.1)$ & 12582 & 12582 \\
\hline Slashdot0811 & $14312(14312)$ & $14333(14337.3)$ & $14312(14312)$ & $14312(14312)$ \\
\hline Slashdot0902 & 15305 & $15334(15338.4)$ & 15305 & 15305 \\
\hline soc-Epinions1 & 15734 & $15742(15746.3)$ & 15734 & 15734 \\
\hline web-BerkStan & 28434 & $30119(30152.4)$ & $28615(28628.9)$ & $28432(28436.1)$ \\
\hline web-Google & $79700(79704.3)$ & $81106(81122.1)$ & 79756(79761.8) & 79699 \\
\hline web-NotDame & $23733(23734.4)$ & $23950(23957.5)$ & 23746 & 23735 \\
\hline web-Stanford & 13198(13199.1) & 14099(14117.6) & $13298(13314.2)$ & $13199(13200.7)$ \\
\hline WikiTalk & 36960 & $\mathbf{3 6 9 6 0}(36961.4)$ & 36960 & 36960 \\
\hline Wiki-Vote & 1116 & 1116 & 1116 & 1116 \\
\hline as-22july06 & 2026 & 2026 & 2026 & 2026 \\
\hline caidaR*Level & $40522(40522.8)$ & $41647(41663.4)$ & $40573(40580.2)$ & 40523 \\
\hline citattionCit*r & 43412 & $44936(44958.3)$ & $43430(43434.3)$ & 43412 \\
\hline cnr-2000 & $22006(22008.5)$ & $22313(22332.1)$ & $22027(22032.6)$ & $22011(22012.2)$ \\
\hline coAuthorCit*r & 33197 & $33790(33818.7)$ & $33343(33358.1)$ & $33197(33197)$ \\
\hline coAuth*DBLP & 43978 & 44640 & 44084(44094.9) & 43978 \\
\hline cond-mat2005 & 650 & 6514(6518.7) & 6531(6537.5) & 6508 \\
\hline coPapersCit*r & $26090(26093.1)$ & 28693(28716.3) & $26989(27008)$ & 26082 \\
\hline coPaperDBLP & $35629(35636.4)$ & 39883(39939.1) & $37060(37090.3)$ & $35597(35599.2)$ \\
\hline eu-2005 & $32292(32300.2)$ & $32812(32831.8)$ & $32322(32330.8)$ & $32284(32287.7)$ \\
\hline in-2004 & 77835(77838.7) & 79016(79036.8) & 77894(77903.2) & $77822(77827.4)$ \\
\hline kron*500log 16 & $14105(14107.1)$ & $14103(14105.2)$ & 14100 & 14100 \\
\hline rgg_n_2_17_s0 & $12378(12385.7)$ & $14705(14745)$ & $13827(13849.4)$ & $12334(12335.9)$ \\
\hline rgg_n_2_19_s0 & $44702(44732.6)$ & $55482(55549.2)$ & $50375(50437.4)$ & $44423(44436.7)$ \\
\hline rgg_n_2_20_s0 & $87222(89920.5)$ & $106700(106754.8)$ & $96649(96703.5)$ & $84708(84729.6)$ \\
\hline rgg_n_2_21_s0 & 180777(181900) & 204553(204696) & 185387(185478) & $162266(162313)$ \\
\hline rgg_n_2_22_s0 & $369858(370458)$ & 393433(393551) & $356868(356945)$ & $312350(312462)$ \\
\hline rgg_n_2_23_s0 & $740096(740750)$ & 757817(758064) & $687552(687945.8)$ & $605278(605592)$ \\
\hline rgg_n_2_24_s0 & $\begin{array}{l}1448537 \\
(1449017.6)\end{array}$ & N/A & $\begin{array}{l}1328020 \\
(1328330)\end{array}$ & $\begin{array}{l}1189207 \\
(1190047.3)\end{array}$ \\
\hline uk-2002 & $\begin{array}{l}1085564 \\
(1085668.2)\end{array}$ & N/A & $\begin{array}{l}1077327 \\
(1077391.7)\end{array}$ & $\begin{array}{l}1075943 \\
(1075951)\end{array}$ \\
\hline 333SP & 676146(676829.7) & ) 698206(698363.9) & $628797(629054)$ & $563599(563738)$ \\
\hline audikw1 & $9496(9510.3)$ & 11389(11416.7) & 10534(10568.5) & $9460(9471.5)$ \\
\hline belgium.osm & $505880(506566)$ & $537618(537898)$ & 476671(476809.9) & $468852(468863)$ \\
\hline cage15 & $518491(518875)$ & $504722(504881)$ & $476574(476878.5)$ & $456062(456340)$ \\
\hline ecology1 & $241117(241345)$ & 269422(269552) & $238052(238250.4)$ & 201498(201669) \\
\hline G_n_pin_pout & $13303(13320.4)$ & $14121(14160.4)$ & $12638(12670.2)$ & $12255(12275.7)$ \\
\hline ldoor & 19798(19807.1) & $24010(24065.9)$ & $21527(21546.4)$ & $19722(19730.9)$ \\
\hline luxem*g.osm & $37753(37754.4)$ & $41229(41333.1)$ & $38421(38444.8)$ & 37751(37752.1) \\
\hline preferAttach* & $8147(8151.2)$ & $8961(8987.4)$ & $8167(8180.1)$ & $8146(8150)$ \\
\hline smallworld & $10756(10767.6)$ & $12744(12769.3)$ & $11733(11770.8)$ & $10451(10456.3)$ \\
\hline wave & $11549(11562.2)$ & $15007(15048.8)$ & 13910(13927.4) & $11619(11675.7)$ \\
\hline
\end{tabular}

Table 5: Experiment results on SNAP and DIMACS10 benchmarks

same solutions on all the instances quickly, but $\mathrm{RLS}_{o}$ and ScBppw are worse. For all standard benchmarks, FastDS has the best performance in terms of solution quality, except slightly worse than $\mathrm{CC}^{2} \mathrm{FS}$ and FastMWDS on UDG benchmark. Over all standard benchmarks, $C^{2} \mathrm{FS}$, FastMWDS and FastDS are essentially better than the other algorithms. We compare the averaged run time of these three algorithms on these benchmarks (Table 4), where the run time of each run of an algorithm is the time to reach the final solution.

\subsection{Results on Massive Benchmarks}

Results on SNAP and DIMACS10 benchmarks are reported in Table 5, and results on Repository benchmarks are reported in Table 6. $\mathrm{CC}^{2} \mathrm{FS}$ is dominated by FastMWDS on all the instances and not reported in Tables 5 and 6 . FastDS performs best for all the massive benchmarks. It obtains the best solutions for 19 (out of 22) SNAP instances, 28 (out of 31) DIMACS10 instances and 63 (out of 65) Repository 


\begin{tabular}{|c|c|c|c|c|}
\hline Instance & $\begin{array}{l}\text { FastMWDS } \\
D_{\min }\left(D_{\text {avg }}\right)\end{array}$ & $\begin{array}{l}\mathrm{RLS}_{0} \\
D_{\min }\left(D_{\text {avg }}\right)\end{array}$ & $\begin{array}{l}\text { ScBppw } \\
D_{\min }\left(D_{\text {avg }}\right)\end{array}$ & $\begin{array}{l}\text { FastDS } \\
D_{\min }\left(D_{\text {avg }}\right)\end{array}$ \\
\hline bn-human-B*1 & $\begin{array}{l}1189968 \\
(1189990)\end{array}$ & $\begin{array}{l}1190728 \\
(1190752)\end{array}$ & $\begin{array}{l}1190244 \\
(1190256)\end{array}$ & $\begin{array}{l}1189874 \\
(1189877.3)\end{array}$ \\
\hline bn-human-B*2 & $\begin{array}{l}1550899 \\
(1550903.4)\end{array}$ & $\begin{array}{l}1551502 \\
(1551519)\end{array}$ & $\begin{array}{l}1551107 \\
(1551119.2)\end{array}$ & $\begin{array}{l}1550863 \\
(1550865.6)\end{array}$ \\
\hline ca-co*-dblp & $35629(35638.7)$ & $39880(39938.6)$ & $37060(37090.3)$ & $35597(35599.2)$ \\
\hline ca-dblp-2012 & 46138 & $46801(46818.5)$ & $46248(46262)$ & 46138 \\
\hline ca-hollyw*d-09 & $51045(51099)$ & $53073(53109.2)$ & $50334(50364.5)$ & $48740(48748.3)$ \\
\hline channel*-b050 & $407609(408691.8)$ & $421739(421938.9)$ & $392298(392409.6)$ & $342645(342886.3)$ \\
\hline dbpedia-link & $1537033(1537076)$ & N/A & $\mathbf{1 5 3 6 6 5 6 ( 1 5 3 6 6 5 7 )}$ & 1536656 \\
\hline delaunay_n22 & $747758(748471.6)$ & 759903(760115.2) & 689061(689178.8) & $640941(641227.5)$ \\
\hline delaunay_n23 & $\begin{array}{l}1516416 \\
(1517199)\end{array}$ & $\begin{array}{l}1519638 \\
(1520217.4)\end{array}$ & $\begin{array}{l}1378243 \\
(1378425.1)\end{array}$ & $\begin{array}{l}\mathbf{1 2 8 8 2 9 7} \\
(1289304.2)\end{array}$ \\
\hline delaunay_n24 & $\begin{array}{l}3044962 \\
(3046047.8)\end{array}$ & N/A & $\begin{array}{l}2756088 \\
(2756512.2)\end{array}$ & $\begin{array}{l}2627137 \\
(2632372)\end{array}$ \\
\hline friendster & $657039(657107.2)$ & $661305(661382.7)$ & $656464(656466.4)$ & 656463 \\
\hline hugebubs- $* 20$ & $\begin{array}{l}6782951 \\
(6783747.3)\end{array}$ & N/A & $\begin{array}{l}5984446 \\
(5984906.2)\end{array}$ & $\begin{array}{l}5926263 \\
(5953412.2)\end{array}$ \\
\hline hugetrace-* 10 & $\begin{array}{l}3850232 \\
(3850634.6)\end{array}$ & N/A & $\begin{array}{l}3392580 \\
(3392947.6)\end{array}$ & $\begin{array}{l}3219420 \\
(3230203.8)\end{array}$ \\
\hline hugetrace- $* 20$ & $\begin{array}{l}5112192 \\
(5113235.7)\end{array}$ & N/A & $\begin{array}{l}4508924 \\
(4509570)\end{array}$ & $\begin{array}{l}4363372 \\
(4380777.8)\end{array}$ \\
\hline inf-euro_osm & $\begin{array}{l}18854928 \\
(18855875.7)\end{array}$ & N/A & $\begin{array}{l}17006807 \\
(17012959.9)\end{array}$ & $\begin{array}{l}17111963 \\
(17156686.5)\end{array}$ \\
\hline inf-germ*_osm & $\begin{array}{l}4237300 \\
(4238386.6)\end{array}$ & N/A & $\begin{array}{l}3846494 \\
(3846917.4)\end{array}$ & $\begin{array}{l}3797838 \\
(3799558.6)\end{array}$ \\
\hline inf-roadNet-CA & 633952(634444.4) & 662093(662374.9) & $595726(595798.5)$ & $586513(586550.2)$ \\
\hline inf-roadNet-PA & $345733(345$ & $369943(37$ & $332079(33214$ & $326934(32695$ \\
\hline inf-road-usa & $\begin{array}{l}8400667 \\
(8401988)\end{array}$ & N/A & $\begin{array}{l}7852540 \\
(7853213.5)\end{array}$ & $\begin{array}{l}7814911 \\
(7818726.7)\end{array}$ \\
\hline rec-dating & $11740(11742.3)$ & $11770(11776.4)$ & $11736(11737.6)$ & $11735(11735.7)$ \\
\hline rec-epinions & 9598 & 9655( & 9599 & 9598 \\
\hline rec-libimset-dir & 12973(12980.4) & 13131(13139.9) & 12955(12956) & 12955(12955.7) \\
\hline rgg_n_2_23_s0 & $740939(741675.6)$ & 757817(758064) & $687552(687946)$ & $605922(606268.9)$ \\
\hline rgg_n_2_24_s0 & $\begin{array}{l}1448992 \\
(1449583.4)\end{array}$ & N/A & $\begin{array}{l}1328020 \\
(1328333.3)\end{array}$ & $\begin{array}{l}1190963 \\
(1194498.1)\end{array}$ \\
\hline rt-retw*-crawl & 75740 & $75816(75822)$ & $\mathbf{7 5 7 4 0}(75740.1)$ & 75740 \\
\hline sc-ldoor & $62474(62486.9)$ & $66589(66625.1)$ & $66244(66309.2)$ & $62411(62422.8)$ \\
\hline sc-msdoor & $19697(19$ & 21408 & 2137 & $19678(19683.3)$ \\
\hline sc-pwtk & 4194( & 5341(5367.7) & 5479 & $4200(420$ \\
\hline sc-rel9 & $123050(125726.7)$ & $143998(148659.7)$ & $124845(129947)$ & $127548(127632.2)$ \\
\hline sc-shipsec1 & $7713(7727.7)$ & $9309(9338.9)$ & $8787(8800.1)$ & $7662(7672.5)$ \\
\hline sc-shipsec5 & 10381(10394.6) & $12560(12577.7)$ & $11873(1189$ & $10300(103$ \\
\hline soc-buzznet & 127 & $130(13$ & 127 & 127 \\
\hline soc-delicious & 55722 & $55969(55984.3)$ & $55726(55727.8)$ & 55722 \\
\hline soc-digg & 66155 & $66641(66671.1)$ & $\mathbf{6 6 1 5 5}(66156.6)$ & 66155 \\
\hline soc-dogster & $26260(26264.5)$ & 26540 & & 26253 \\
\hline socfb-A-anon & 201774(201793.6) & 203194(203218.6) & $1690.5)$ & 201690 \\
\hline socfb-B-anon & 187031(187036) & $187829(187850.7)$ & $\mathbf{1 8 7 0 3 0}(187030.1)$ & 187030 \\
\hline socfb-uci-uni & $865675(865675.1)$ & N/A & 865675 & 865675 \\
\hline soc-flickr & 98062(98062.5) & $98640(98658.7)$ & 98063(98064.9) & 98062 \\
\hline soc-flickr-und & 295773(295790.9) & 296979(296991.9) & $295702(295705.1)$ & 295700 \\
\hline soc-flixster & 91019 & $91127(91131.9)$ & 91019 & 91019 \\
\hline soc-FourSq* & 60982(60985.8) & 61301(61316.3) & 60979 & 60979 \\
\hline soc-lastfm & 67226 & 67264 & 67226 & 67226 \\
\hline soc-livejour* & $803448(803626.7)$ & $815040(815178.6)$ & 793988(793997.1) & 793887(793887.9) \\
\hline soc-live* groups & $\begin{array}{l}1072271 \\
(1072315.9)\end{array}$ & $\begin{array}{l}1074058 \\
(1074107.7)\end{array}$ & $\begin{array}{l}1071123 \\
(1071124)\end{array}$ & 1071123 \\
\hline soc-LiveMocha & 1424 & $1475(1482)$ & 1424 & 1424 \\
\hline soc-ljour*2008 & $\begin{array}{l}1015864 \\
(1016080.1)\end{array}$ & $\begin{array}{l}1025928 \\
(1026043.5)\end{array}$ & $\begin{array}{l}1005983 \\
(1005988.6)\end{array}$ & 1005858 \\
\hline soc-orkut & 120334(120416.1) & $124516(124581.3)$ & 111087(111117.8) & $110547(110585.8)$ \\
\hline soc-orkut-dir & 100971(101010.1) & $104751(10486$ & $93974(94013.3)$ & $93630(93644$ \\
\hline soc-pokec & 213149(213242) & $221665(221816.5)$ & 207385(207397) & $207308(207312.3)$ \\
\hline soc-sinaweibo & 201328 & N/A & 201328 & 201328 \\
\hline soc-twit*-higgs & $14751(14763.3)$ & $15235(15257.8)$ & $\mathbf{1 4 6 8 9}(14690.6)$ & $14689(14689.4)$ \\
\hline soc-youtube & 89732 & $90525(90558.2)$ & 89734(89735.3) & 89732 \\
\hline soc-yout*-snap & 213167(213174.6) & 214014(214041.8) & $\mathbf{2 1 3 1 2 2}(213123.3)$ & 213122 \\
\hline tech-as-skitter & 183324(183378.8) & $186588(186648.6)$ & $181852(181869.8)$ & $181717(181717$ \\
\hline tech-ip & $160(162.9)$ & $174(176.1)$ & $155(157.9)$ & $154(154.8)$ \\
\hline twitter_mpi & $566378(566401.4)$ & $567050(567079.3)$ & $\mathbf{5 6 6 3 1 5}(566315.5)$ & $566315(566315)$ \\
\hline web-arab*05 & 16901(16901.3) & 17176(17191.3) & $16907(16909.5)$ & $16901(16901.4)$ \\
\hline & 278191(278204.7) & $279702(279727.1)$ & $277849(277850.4)$ & 277847 \\
\hline web-it-2004 & 32997 & $33085(33093.6)$ & $32998(32999.3)$ & 32997 \\
\hline web-uk-2005 & 1421 & 1421 & 1421 & 1421 \\
\hline web-wik*_link & $338691(338753.2)$ & $339651(339679)$ & $336688(336690.9)$ & $336682(336682.9)$ \\
\hline web-wik*2009 & $348003(348024.5)$ & $352396(352492.8)$ & $346676(346682.5)$ & $346581(346584)$ \\
\hline web-wiki*grow & $117626(117663)$ & $118917(118967.5)$ & $116817(116818.7)$ & $116814(116814.6)$ \\
\hline wikip*_link_en & $\begin{array}{l}23995929 \\
(23995935.9)\end{array}$ & N/A & 23995924 & 23995924 \\
\hline
\end{tabular}

Table 6: Experiment results on Repository benchmark

instances. On average, the best solutions found by FastDS have 1320 and 23732 fewer vertices than the strongest com-

\begin{tabular}{lllllll}
\hline benchmark & \#total & $\begin{array}{l}\text { CC }^{2} \text { FS } \\
\text { \#min }\end{array}$ & $\begin{array}{l}\text { FastMWDS } \\
\text { \#min }\end{array}$ & $\begin{array}{l}\text { RLS }_{0} \\
\text { \#min }\end{array}$ & $\begin{array}{l}\text { ScBppw } \\
\text { \#min }\end{array}$ & $\begin{array}{l}\text { FastDS } \\
\text { \#min }\end{array}$ \\
\hline UDG & 12 & $\mathbf{1 2}$ & $\mathbf{1 2}$ & 8 & 2 & 11 \\
T1 & 48 & 43 & 42 & 19 & 7 & $\mathbf{4 7}$ \\
DIMACS & 61 & $\mathbf{6 0}$ & $\mathbf{6 0}$ & 51 & 45 & $\mathbf{6 0}$ \\
BHOSLIB & 31 & 29 & 28 & 2 & 0 & $\mathbf{3 1}$ \\
\hline SNAP & 22 & 10 & 15 & 7 & 11 & $\mathbf{1 9}$ \\
DIMACS10 & 31 & 1 & 9 & 1 & 2 & $\mathbf{2 8}$ \\
Repository & 65 & 5 & 17 & 1 & 19 & $\mathbf{6 3}$ \\
\hline
\end{tabular}

Table 7: Summary results of all benchmarks. We report for each algorithm the number of instances (or families) where it finds the best $D_{\min }$ among the algorithms in experiments.

\begin{tabular}{llll}
\hline Benchmark & fix_ratio & ctime_rules & ctime_no_rules \\
\hline SNAP & $28.59 \%$ & $0.98 \mathrm{~s}$ & $1.07 \mathrm{~s}$ \\
DIMACS10 & $7.21 \%$ & $4.57 \mathrm{~s}$ & $4.42 \mathrm{~s}$ \\
Repository & $23.82 \%$ & $35.98 \mathrm{~s}$ & $38.43 \mathrm{~s}$ \\
\hline
\end{tabular}

Table 8: The fix ratio of the inference rules and construction time with and without rules

petitor FastMWDS for SNAP and DIMACS10 benchmarks, and have 14150 fewer vertices than the strongest competitor ScBppw for Repository benchmark. Table 7 summarizes the comparison results on all the benchmarks.

\subsection{Analysis of Underlying Ideas}

We calculate the portion of vertices fixed by inference rules among all vertices, and compare the run time of the construction procedure with and without the rules (Table 8). The inference rules are effective on the massive benchmarks with little overhead. FastDS without the rules degrades obviously on the massive benchmarks. It gets worse solutions on half Repository instances. However, the rules have little impact on the standard benchmarks.

We also test a variant of FastDS (denoted as FastDS_g1) that uses the one-goal framework (targeting size of $(k-1)$ after finding a $k$ sized solution) to replace our two-goal framework. FastDS finds better solutions than FastDS_g1 on 12, 48 and 31 instances for UDG, T1 and BHOSLIB benchmarks respectively, and worse on none of the instances. For massive benchmarks, FastDS finds better solutions on 11, 25 and 39 instances for SNAP, DIMACS10 and Repository respectively, while worse than FastDS_g1 only on 3 and 9 instances in DIMACS10 and Repository benchmarks respectively.

\section{Conclusions}

We proposed a two-goal local search framework for MinDS and proposed three inference rules. The resulting algorithm FastDS is robust and efficient on standard benchmarks and massive benchmarks, and significantly outperforms state-ofthe-art algorithms on massive benchmarks. We would like to study the ideas for other subset problems.

\section{Acknowledgements}

This work is supported by Beijing Academy of Artificial Intelligence (BAAI), Youth Innovation Promotion Association, Chinese Academy of Sciences [No. 2017150], and NSFC Grant 61806050. 


\section{References}

[Abu-Khzam et al., 2017] Faisal N. Abu-Khzam, Shaowei Cai, Judith Egan, Peter Shaw, and Kai Wang. Turbo-charging dominating set with an FPT subroutine: Further improvements and experimental analysis. In Proceedings of TAMC 2017, pages 5970, 2017.

[Bouamama and Blum, 2016] Salim Bouamama and Christian Blum. A hybrid algorithmic model for the minimum weight dominating set problem. Simulation Modelling Practice and Theory, 64:57-68, 2016.

[Cai and Lin, 2016] Shaowei Cai and Jinkun Lin. Fast solving maximum weight clique problem in massive graphs. In Proceedings of IJCAI 2016, pages 568-574, 2016.

[Cai et al., 2011] Shaowei Cai, Kaile Su, and Abdul Sattar. Local search with edge weighting and configuration checking heuristics for minimum vertex cover. Artif. Intell., 175(9-10):1672-1696, 2011.

[Cai et al., 2013] Shaowei Cai, Kaile Su, Chuan Luo, and Abdul Sattar. Numvc: An efficient local search algorithm for minimum vertex cover. J. Artif. Intell. Res., 46:687-716, 2013.

[Cai et al., 2017] Shaowei Cai, Jinkun Lin, and Chuan Luo. Finding A small vertex cover in massive sparse graphs: Construct, local search, and preprocess. J. Artif. Intell. Res., 59:463-494, 2017.

[Cai, 2015] Shaowei Cai. Balance between complexity and quality: Local search for minimum vertex cover in massive graphs. In Proceedings of IJCAI 2015, pages 747-753, 2015.

[Chalupa, 2018] David Chalupa. An order-based algorithm for minimum dominating set with application in graph mining. Inf. Sci., 426:101-116, 2018.

[Chaurasia and Singh, 2015] Sachchida Nand Chaurasia and Alok Singh. A hybrid evolutionary algorithm with guided mutation for minimum weight dominating set. Appl. Intell., 43(3):512-529, 2015.

[Fan et al., 2019] Yi Fan, Yongxuan Lai, Chengqian Li, Nan Li, Zongjie Ma, Jun Zhou, Longin Jan Latecki, and Kaile Su. Efficient local search for minimum dominating sets in large graphs. In Proceedings of DASFAA 2019, pages 211-228, 2019.

[Gao et al., 2015] Chao Gao, Xin Yao, Thomas Weise, and Jinlong Li. An efficient local search heuristic with row weighting for the unicost set covering problem. European Journal of Operational Research, 246(3):750-761, 2015.

[Gao et al., 2018] Jian Gao, Jiejiang Chen, Minghao Yin, Rong Chen, and Yiyuan Wang. An exact algorithm for maximum kplexes in massive graphs. In Proceedings of IJCAI 2018, pages 1449-1455, 2018.

[Gast et al., 2015] Mikael Gast, Mathias Hauptmann, and Marek Karpinski. Inapproximability of dominating set on power law graphs. Theor. Comput. Sci., 562:436-452, 2015.

[Hedar and Ismail, 2010] Abdel-Rahman Hedar and Rashad Ismail. Hybrid genetic algorithm for minimum dominating set problem. In Proceedings of ICCSA 2010, pages 457-467, 2010.

[Jovanovic et al., 2010] Raka Jovanovic, Milan Tuba, and Dana Simian. Ant colony optimization applied to minimum weight dominating set problem. In 12th international conference on $\mathrm{Au}$ tomatic control, modelling \& simulation, pages 322-326, 2010.

[Karthik C. S. et al., 2018] Karthik C. S., Bundit Laekhanukit, and Pasin Manurangsi. On the parameterized complexity of approximating dominating set. In Proceedings of STOC 2018, pages 1283-1296, 2018.
[Li et al., 2019] Ruizhi Li, Shuli Hu, Huan Liu, Ruiting Li, Dantong Ouyang, and Minghao Yin. Multi-start local search algorithm for the minimum connected dominating set problems. Mathematics, 7(12):1173, 2019.

[Lin et al., 2016] Geng Lin, Wenxing Zhu, and M. Montaz Ali. An effective hybrid memetic algorithm for the minimum weight dominating set problem. IEEE Trans. Evolutionary Computation, 20(6):892-907, 2016.

[Lin et al., 2017] Jinkun Lin, Shaowei Cai, Chuan Luo, and Kaile $\mathrm{Su}$. A reduction based method for coloring very large graphs. In Proceedings of IJCAI 2017, pages 517-523, 2017.

[Musliu, 2006] Nysret Musliu. Local search algorithm for unicost set covering problem. In International Conference on Industrial, Engineering and Other Applications of Applied Intelligent Systems, pages 302-311. Springer, 2006.

[Potluri and Singh, 2011] Anupama Potluri and Alok Singh. Two hybrid meta-heuristic approaches for minimum dominating set problem. In Proceedings of SEMCCO 2011, pages 97-104, 2011.

[Potluri and Singh, 2013] Anupama Potluri and Alok Singh. Hybrid metaheuristic algorithms for minimum weight dominating set. Appl. Soft Comput., 13(1):76-88, 2013.

[Raz and Safra, 1997] Ran Raz and Shmuel Safra. A sub-constant error-probability low-degree test, and a sub-constant errorprobability PCP characterization of NP. In Proceedings of STOC 1997, pages 475-484, 1997.

[Richter et al., 2007] Silvia Richter, Malte Helmert, and Charles Gretton. A stochastic local search approach to vertex cover. In Proceedings of KI 2007, pages 412-426, 2007.

[Rossi and Ahmed, 2015] Ryan A. Rossi and Nesreen K. Ahmed. The network data repository with interactive graph analytics and visualization. In Proceedings of AAAI 2015, pages 4292-4293, 2015.

[Sanchis, 2002] Laura A. Sanchis. Experimental analysis of heuristic algorithms for the dominating set problem. Algorithmica, 33(1):3-18, 2002.

[van Rooij and Bodlaender, 2011] Johan M. M. van Rooij and Hans L. Bodlaender. Exact algorithms for dominating set. Discrete Applied Mathematics, 159(17):2147-2164, 2011.

[Verma et al., 2015] Anurag Verma, Austin Buchanan, and Sergiy Butenko. Solving the maximum clique and vertex coloring problems on very large sparse networks. INFORMS Journal on Computing, 27(1):164-177, 2015.

[Wang et al., 2009] Feng Wang, Erika Camacho, and Kuai Xu. Positive influence dominating set in online social networks. In Proceedings of COCOA 2009, pages 313-321, 2009.

[Wang et al., 2017] Yiyuan Wang, Shaowei Cai, and Minghao Yin. Local search for minimum weight dominating set with two-level configuration checking and frequency based scoring function. $J$. Artif. Intell. Res., 58:267-295, 2017.

[Wang et al., 2018] Yiyuan Wang, Shaowei Cai, Jiejiang Chen, and Minghao Yin. A fast local search algorithm for minimum weight dominating set problem on massive graphs. In Proceedings of IJCAI 2018, pages 1514-1522, 2018.

[Xu et al., 2007] Ke Xu, Frédéric Boussemart, Fred Hemery, and Christophe Lecoutre. Random constraint satisfaction: Easy generation of hard (satisfiable) instances. Artificial intelligence, 171(8-9):514-534, 2007. 